\title{
Notes from the Editor
}

The present issue of the JOURNAL OF SOCIOCYBERNETICS is the last edition under my responsibility. In January 2015 the quite recently elected board of the ISA-Research Committee 51 will take up its work and Fabio Giglietto, Professor at the Department of Communication and Human Studies of the University of Urbino "Carlo Bo" (http://www.uniurb.it) and research fellow of the "Center for Sociocybernetics Studies (http://www.sociocybernetics.eu)", will commence as the new journal editor. During the last four years Fabio Giglietto, already was a member of the editorial board of our Journal. I wish him and the new board continued success and all the best for the upcoming period.

The current edition includes articles applying several theoretical aspects of complexity analysis on different empirical cases. In their article "Reflections on the Complexity of Ancient Social Heterarchies: Toward New Models of Social Self-Organization in Pre-Hispanic Colombia" Nathalie Mezza-Garcia, Tom Froese and Nelson Fernández face the limitations which hierarchical and centrally controlled systems have in their information processes with respect to manage large-scale crisis and challenges. With reference to historical examples in pre-Hispanic Colombia, specifically the cultures of the Zenú, the Muiscas and the Tayronas, the authors propose that creating and analyzing computer models of their heterarchically and decentralized processes of management could provide a broader perspective on the possibilities of self-organized political systems.

In his article "The Paradox of Social Ties after the ICT Revolution: A Second-Order Observation" Saburo Akahori explores what kinds of distinctions are used when the change of social systems is observed. His analysis refers on the question of significance of social ties in Japan, which has repeatedly been emphasized in recent years. One example is the frequency of use of the Japanese word kizuna which means bond. It sounds odd because conventionally kizuna indicates intimate, continuous relationships, not temporary relationships. Even though the word kizuna means strong ties, now it also implies weak ties. Here the author asks for the reason why the strange usage of the word kizuna has become acceptable.

Patricia E. Almaguer-Kalixto, José A. Amozurrutia, Chaime Marcuello Servós present in their paper "Policy Processes as Complex Systems: The case of Mesoamerican Sustainable Development Initiative" a research methodology for analyzing policy processes that are defined at the global level but implemented locally. The interrelations between these two levels pose great conceptual challenges in explaining the changes, transformations and continuations occurring in this complex process based on empirical information. Understanding the policy process as a complex system, the paper proposes analyzing macro, meso and micro levels as subsystems of the total process, identifying the interre- 
lations between policy action, actors and discourses. The paper takes the example of the Mesoamerican Sustainable Development Initiative (MSDI) of the Puebla Panama Plan (PPP), a regional integration plan for a new 'Mesoamerica' that originally included the seven Central American countries and the southern states of Mexico.

In her paper "Sustainable Technology Assessment and Sustainable Scenarios of Techno Social Phenomena" Michiko Amemiya-Ramírez describes sustainable technology as a technological subsystem with marginal or no negative impacts on other technological systems, as well as the environment, the society and the economy. To identify such technologies it is necessary to describe their behavior and their present and future interactions with those systems. Due to social dynamics, a complete assessment to identify sustainable technologies requires a hard systems analysis and a soft system analysis. A hard system analysis is useful to assess the interactions, behavior and characteristics of the technology quantitatively. A soft system analysis is convenient to describe other characteristics and interactions through qualitative and non measurable characteristics.

For further issues of the JOURNAL OF SOCIOCYBERNETICS we invite scholars who have their background in the field of systems theory, sociocybernetics, information- and communication science and who apply this for studying various social phenomena regarding their complexity and dynamics, to submit articles for publication in the JOURNAL OF SOCIOCYBERNETICS. For submitting articles authors need to register with the journal prior to submitting. People who want to register have the option to register as a reader or as an author. Every reader or author can register by themselve using the journal's website. After clicking the register item they will be guided through the registration process. After registration they will be able to login by username and password and then authors may submit their papers. The system will immediately confirm the submission and will automatically trigger the review process. Authors will get an email with a URL that will enable them to track its progress through the editorial process once they are logged in. We recommend to review the "About the Journal" page for the journal's policies, as well as the "Author Guidelines".

Michael Paetau (Editor) 